\title{
EQUIVALENCE OF CONTROL SYSTEMS WITH LINEAR SYSTEMS ON LIE GROUPS AND HOMOGENEOUS SPACES
}

\author{
PHILIPPE JOUAN $^{1}$
}

\begin{abstract}
The aim of this paper is to prove that a control affine system on a manifold is equivalent by diffeomorphism to a linear system on a Lie group or a homogeneous space if and only if the vector fields of the system are complete and generate a finite dimensional Lie algebra.

A vector field on a connected Lie group is linear if its flow is a one parameter group of automorphisms. An affine vector field is obtained by adding a left invariant one. Its projection on a homogeneous space, whenever it exists, is still called affine.

Affine vector fields on homogeneous spaces can be characterized by their Lie brackets with the projections of right invariant vector fields.

A linear system on a homogeneous space is a system whose drift part is affine and whose controlled part is invariant.

The main result is based on a general theorem on finite dimensional algebras generated by complete vector fields, closely related to a theorem of Palais, and which has its own interest. The present proof makes use of geometric control theory arguments.
\end{abstract}

Mathematics Subject Classification. 17B66, 57S15, 57S20, 93B17, 93B29.

Received December 5, 2008.

Published online July 31, 2009.

\section{INTRODUCTION}

The main purpose of this paper is to characterize the class of control systems

$$
\dot{p}=f(p)+\sum_{j=1}^{m} u_{j} g_{j}(p)
$$

which are globally diffeomorphic to a linear system on a Lie group or a homogeneous space. They turn out to be the systems whose vector fields are complete and generate a finite dimensional Lie algebra.

We say that a vector field on a connected Lie group is linear if its flow is a one parameter group of automorphisms. Linear vector fields on Lie groups are nothing else than the so-called infinitesimal automorphisms in the Lie group literature (see for instance [3]). They were first considered in a control theory context by Markus,

Keywords and phrases. Lie groups, homogeneous spaces, linear systems, complete vector field, finite dimensional Lie algebra.

1 LMRS, CNRS UMR 6085, Université de Rouen, avenue de l'Université, BP 12, 76801 Saint-Étienne-du-Rouvray, France. Philippe.Jouan@univ-rouen.fr 
on matrix Lie groups (see [9]), and then in the general case by Ayala and Tirao (see [2]). They are the natural extension to Lie groups of the linear fields on vector spaces and, for this reason, are still called linear.

There is another way, of course equivalent, to define linear vector fields on a connected Lie group $G$. Let us denote by $\mathfrak{g}$ its Lie algebra, that is the set of right invariant vector fields. Then a vector field $\mathcal{X}$ is linear if and only if

$$
\forall Y \in \mathfrak{g} \quad[\mathcal{X}, Y] \in \mathfrak{g}
$$

and moreover satisfies $\mathcal{X}(e)=0$, where $e$ stands for the identity of $G$. In case where $\mathcal{X}$ satisfies condition (1.2) but not $\mathcal{X}(e)=0$ it will be said affine (it is in that case equal to the sum of a linear vector field and a left invariant one). This second definition was introduced in [2] for affine vector fields (on Lie groups). Its interest is twofold. On the one hand it does not require the knowledge of the flow of $\mathcal{X}$. On the other one it can be extended to homogeneous spaces.

An affine vector field on a homogeneous space $G / H$ is the projection, if it exists, of an affine vector field on $G$. Theorem 4.1 provides a characterization of affine vector fields on homogeneous spaces in terms of Lie brackets with the invariant ones, similar to (1.2).

A system defined on a homogeneous space,

$$
\dot{x}=F(x)+\sum_{j=1}^{m} u_{j} Y_{j}(x),
$$

is called linear if the field $F$ is affine and the $Y_{j}$ 's invariant. Linear systems on Lie groups and invariant systems appear as particular cases of this general setting.

The extension of affine vector fields and linear systems to homogeneous spaces is motivated by the fact that the class of systems diffeomorphic to a linear one on a Lie group is rather restrictive, while the class of systems diffeomorphic to linear systems on homogeneous spaces is much wider. More accurately we have (Thm. 6.1, Sect. 6):

We assume the family $\left\{f, g_{1}, \ldots, g_{m}\right\}$ to be transitive. Then system (1.1) is diffeomorphic to a linear system on a Lie group or a homogeneous space if and only if the vector fields $f, g_{1}, \ldots, g_{m}$ are complete and generate a finite dimensional Lie algebra.

In this statement, the transitivity assumption means that there is only one orbit, equal to the state space, under the action of the system. This is not really a limitation since by the Orbit theorem (see Sect. 2) we know that we can always consider the restriction of the system to the orbit through a given point.

The proof makes appeal to Theorem 5.1 (Sect. 5):

Let $\Gamma$ be a transitive family of vector fields on a connected manifold $M$. If all the vector fields belonging to $\Gamma$ are complete, and if $\Gamma$ generates a finite dimensional Lie algebra $\mathcal{L}(\Gamma)$, then $M$ is diffeomorphic to a homogeneous space $G / H$, where $G$ is a simply connected Lie group whose Lie algebra is isomorphic to $\mathcal{L}(\Gamma)$. The tangent mapping of this diffeomorphism induces an isomorphism from $\mathcal{L}(\Gamma)$ onto the Lie algebra of invariant vector fields on $G / H$, and all the vector fields belonging to $\mathcal{L}(\Gamma)$ are complete.

Theorem 5.1 is very closely related to a theorem of Palais ([10], Thm. III, p. 95), but the new proof given here uses control theory ideas like transitivity, rank condition, normal accessibility, Sussmann's Orbit Theorem. An important consequence of Theorem 5.1 is that the families of vector fields under consideration are Lie determined.

Thus linear systems on Lie groups and homogeneous spaces appear as models for a wide class of systems, and will certainly play an important role in studying topics such as controllability, stabilization, optimal control and observability. Some results about controllability and observability of linear systems on Lie groups are already known, see $[1,2,4,6]$.

The paper is organized as follows.

In Section 2 the definitions and facts from control theory used in the sequel are recalled.

In Section 3 the various definitions of linear and affine vector fields on Lie groups are stated, and their equivalence proved (see Thm. 3.1), as well as their properties, in particular their completeness which is one 
of the main ingredients used herein. Some of the proofs can be found in the literature (for instance [2-4]) and are in that case quoted, but to the author's knowledge, Theorem 3.1 is nowhere completely proved.

In Section 4 affine vector fields on homogeneous spaces are introduced, and characterized by their Lie brackets with the projections of right invariant vector fields (Thm. 4.1).

Section 5 is devoted to the previously mentioned Theorem 5.1, and to a corollary (see Cor. 5.1) where the transitivity assumption is relaxed.

The main result, Theorem 6.1, is stated and proved in Section 6, which is ended by Corollary 6.1 where systems diffeomorphic to a linear system on a simply connected Lie group are characterized.

Section 7 begins by some examples of linear vector fields. Then we consider a well known system and show that it is equivalent to a linear one on a homogeneous space of the group Heisenberg. The equivalence is computed.

Throughout the paper (in fact from Sect. 5) the vector fields under consideration are only assumed to be $\mathcal{C}^{k}$, $k \geq 1$, because the class of differentiability does not matter. The important properties are the completeness of the vector fields and the fact that they generate a finite dimensional Lie algebra.

On the other hand the simply connected spaces will be assumed to be connected. However this definition of simply connectedness, that can be found for instance in [5], is not universal and will be recalled in some statements.

\section{Preliminaries}

In this section some standard definitions and facts from control theory are reviewed.

Let $\Gamma=\left\{g_{i} ; i \in I\right\}$ be a family of $\mathcal{C}^{k}$ vector fields on a connected $\mathcal{C}^{k+1}$ manifold $M$, with $k \geq 1$ (the $g_{i}$ 's are not required to be complete).

Let us denote by $\left(\gamma_{t}^{i}\right)$ the flow of $g_{i}$. The orbit of $\Gamma$ through a point $p \in M$ is the set of points $q$ for which there exist vector fields $g_{i_{1}}, \ldots, g_{i_{r}} \in \Gamma$, and real numbers $t_{1}, \ldots, t_{r}$ such that

$$
\gamma_{t_{r}}^{i_{r}} \circ \ldots \circ \gamma_{t_{1}}^{i_{1}}(p)
$$

is defined and equal to $q$. Let us recall the Sussmann's orbit theorem:

Orbit Theorem. The orbit of $\Gamma$ through each point $p$ of $M$ is a connected immersed submanifold of $M$.

In the original proof of Sussmann the vector fields are assumed to be $\mathcal{C}^{\infty}$ (see [11]), but a proof for $\mathcal{C}^{k}$ vector fields, with $k \geq 1$, can be found in [7].

This family of vector fields is said to be transitive if the orbit through each point $p$ of $M$ is equal to $M$, that is if $M$ is the only orbit of $\Gamma$.

Let $V^{k}(M)$ stand for the space of $\mathcal{C}^{k}$ vector fields on $M$. It is not a Lie algebra whenever $k<+\infty$. But it may happen that all the Lie brackets of elements of $\Gamma$ of all finite lengths exist and are also $\mathcal{C}^{k}$. In that case the subspace of $V^{k}(M)$ spanned by these Lie brackets is a Lie algebra and we will say that the family $\Gamma$ generates a Lie algebra. This last will be denoted by $\mathcal{L}(\Gamma)$.

Let us assume that $\Gamma$ generates a Lie algebra, and let us consider the rank of $\Gamma$ at each point $p \in M$, that is the dimension of the subspace of $T_{p} M$, the tangent space to $M$ at $p$, spanned by the vectors $\gamma(p), \gamma \in \mathcal{L}(\Gamma)$. The so-called rank condition asserts that the family $\Gamma$ is transitive as soon as its rank is maximum, hence equal to $\operatorname{dim} M$, at each point.

To finish let us recall the definition of Lie-determined systems (see for instance [7]): the family $\Gamma$ is said to be Lie-determined if at each point $p \in M$, the rank of $\Gamma$ at $p$ is equal to the dimension of the orbit of $\Gamma$ through $p$. 


\section{LiNEAR AND AFFINE VECTOR FIELDS ON LIE GROUPS}

Let $G$ be a connected Lie group, and $\mathfrak{g}$ its Lie algebra, that is the set of right invariant vector fields. The set of analytic vector fields on $G$ is denoted by $V^{\omega}(G)$, and the normalizer of $\mathfrak{g}$ in $V^{\omega}(G)$ is by definition

$$
\mathcal{N}=\operatorname{norm}_{V^{\omega}(G)} \mathfrak{g}=\left\{F \in V^{\omega}(G) ; \forall Y \in \mathfrak{g} \quad[F, Y] \in \mathfrak{g}\right\}
$$

Definition 3.1. A vector field $F$ on $G$ is said to be affine if it belongs to $\mathcal{N}$.

Such a vector field $F$ is said to be linear if it moreover verifies $F(e)=0$, where $e$ stands for the identity of $G$.

In other words the restriction of $a d(F)$ to $\mathfrak{g}$, also denoted by $\operatorname{ad}(F)$, is a derivation of $\mathfrak{g}$. From the Jacobi identity, it is clear that $\mathcal{N}$ is a Lie subalgebra of $V^{\omega}(G)$, and that the mapping $F \longmapsto \operatorname{ad}(F)$ is a Lie algebra morphism from $\mathcal{N}$ into $\mathcal{D}(\mathfrak{g})$, the set of derivations of $\mathfrak{g}$.

Proposition 3.1 (see [2]). The kernel of the mapping $F \longmapsto a d(F)$ is the set of left invariant vector fields. An affine vector field $F$ can be uniquely decomposed into a sum

$$
F=\mathcal{X}+Z
$$

where $\mathcal{X}$ is linear and $Z$ left invariant.

This proposition is no longer true whenever the group $G$ is not connected.

Proof. Let $Z$ be an affine vector field whose bracket with any element of $\mathfrak{g}$ vanishes. Its flow $z_{t}$ commutes with the one of any $Y \in \mathfrak{g}$. This writes

$$
\forall x \in G \quad z_{t}(\exp (s Y) x)=\exp (s Y) z_{t}(x)
$$

for all $t, s \in \mathbb{R}$ for which this makes sense. Fix $x \in G$. There exist $Y_{1} \ldots Y_{k} \in \mathfrak{g}$ such that

$$
x=\exp \left(Y_{1}\right) \ldots \exp \left(Y_{k}\right),
$$

thanks to the connectedness of $G$. Therefore

$$
z_{t}(x)=z_{t}\left(\exp \left(Y_{1}\right) \ldots \exp \left(Y_{k}\right)\right)=\exp \left(Y_{1}\right) \ldots \exp \left(Y_{k}\right) z_{t}(e)=x z_{t}(e)
$$

for $t$ sufficiently small, and

$$
\begin{aligned}
Z_{x} & =\frac{\mathrm{d}}{\mathrm{d} t} \mid t=0 \\
& =\frac{\mathrm{d}}{\mathrm{d} t} z_{t=0}(x) \\
& =T_{e} L_{x} \cdot Z_{e}(e)
\end{aligned}
$$

where $T_{e} L_{x}$ stands for the tangent mapping at the identity $e$ of the left translation $L_{x}$. This proves that $Z$ is left invariant and, the converse being obvious, since the bracket of a left-invariant vector field with a right-invariant one vanishes, the first part of the proposition.

For the second one let $Z$ be the left invariant vector field defined by $Z_{e}=F(e)$. Then the vector field $\mathcal{X}=F-Z$ is clearly linear.

Theorem 3.1. Let $\mathcal{X}$ be a vector field on a connected Lie group $G$. The following conditions are equivalent:

(1) $\mathcal{X}$ is linear;

(2) the flow of $\mathcal{X}$ is a one parameter group of automorphisms of $G$;

(3) $\mathcal{X}$ verifies

$$
\forall x, x^{\prime} \in G \quad \mathcal{X}_{x x^{\prime}}=T L_{x} \cdot \mathcal{X}_{x^{\prime}}+T R_{x^{\prime}} \cdot \mathcal{X}_{x}
$$

The second item implies that a linear vector field on a connected Lie group is complete. 
Proof. (1) $\Rightarrow(3)$. The vector field $\mathcal{X}$ is linear, hence for all $Y \in \mathfrak{g}$, the Lie bracket $[\mathcal{X}, Y]$ is right invariant. Therefore we have

$$
\forall x \in G \quad[\mathcal{X}, Y]=R_{x *}[\mathcal{X}, Y]=\left[R_{x *} \mathcal{X}, R_{x *} Y\right]=\left[R_{x *} \mathcal{X}, Y\right] .
$$

This proves firstly that $\left[R_{x *} \mathcal{X}, Y\right]$ is right invariant for all $Y \in \mathfrak{g}$, hence that the vector field $R_{x *} \mathcal{X}$ is affine, and secondly that $\operatorname{ad}\left(R_{x *} \mathcal{X}\right)=\operatorname{ad}(\mathcal{X})$ hence following Proposition 3.1 that

$$
R_{x *} \mathcal{X}=\mathcal{X}+Z
$$

where $Z$ is left invariant. This last is characterized by

$$
Z_{e}=\left(R_{x *} \mathcal{X}\right)_{e}=T R_{x} \cdot \mathcal{X}_{x^{-1}}
$$

hence for all $x^{\prime} \in G$

$$
Z_{x^{\prime}}=T L_{x^{\prime}} T R_{x} \cdot \mathcal{X}_{x^{-1}}=T R_{x} T L_{x^{\prime}} \cdot \mathcal{X}_{x^{-1}} .
$$

Considering $\left(R_{x *} \mathcal{X}\right)_{x^{\prime}}=T R_{x} \cdot \mathcal{X}_{x^{\prime} x^{-1}}$, equality (3.2) evaluated at the point $x^{\prime}$ becomes

$$
T R_{x} \cdot \mathcal{X}_{x^{\prime} x^{-1}}=\mathcal{X}_{x^{\prime}}+T R_{x} T L_{x^{\prime}} \cdot \mathcal{X}_{x^{-1}}
$$

It remains to apply $T R_{x^{-1}}$ to obtain

$$
\mathcal{X}_{x^{\prime} x^{-1}}=T R_{x^{-1}} \cdot \mathcal{X}_{x^{\prime}}+T L_{x^{\prime}} \cdot \mathcal{X}_{x^{-1}}
$$

and to replace $x^{-1}$ by $x$ to obtain equality (3.1).

$(3) \Rightarrow(2)$ (see also [3]). Let us denote by $\varphi_{t}$ the flow of $\mathcal{X}$, defined on a domain of $\mathbb{R} \times G$. The curve

$$
t \longmapsto \varphi_{t}(x) \varphi_{t}\left(x^{\prime}\right)
$$

is defined on an open interval containing 0 , and takes the value $x x^{\prime}$ at $t=0$. Moreover

$$
\begin{aligned}
\frac{\mathrm{d}}{\mathrm{d} t} \varphi_{t}(x) \varphi_{t}\left(x^{\prime}\right) & =T L_{\varphi_{t}(x)} \cdot \mathcal{X}_{\varphi_{t}\left(x^{\prime}\right)}+T R_{\varphi_{t}\left(x^{\prime}\right)} \cdot \mathcal{X}_{\varphi_{t}(x)} \\
& =\mathcal{X}_{\varphi_{t}(x) \varphi_{t}\left(x^{\prime}\right)} .
\end{aligned}
$$

This proves that

$$
\varphi_{t}(x) \varphi_{t}\left(x^{\prime}\right)=\varphi_{t}\left(x x^{\prime}\right)
$$

as soon as the left-hand side exists. It remains to show that $\mathcal{X}$ is complete. To begin with, notice that equality (3.1), evaluated at $x=x^{\prime}=e$, implies $\mathcal{X}_{e}=0$.

Let $x \in G$ and $t \in \mathbb{R}$. Since $\mathcal{X}_{e}$ vanishes, $\varphi_{t}$ is defined on an open neighborhood $V_{t}$ of $e$. The group $G$ being connected, it is generated by this neighborhood. Therefore there exist $x_{1}, \ldots, x_{n} \in V_{t}$ such that $x=x_{1} \ldots x_{n}$, and

$$
\varphi_{t}(x)=\varphi_{t}\left(x_{1} \ldots x_{n}\right)=\varphi_{t}\left(x_{1}\right) \ldots \varphi_{t}\left(x_{n}\right)
$$

is well defined. This proves that $\mathcal{X}$ is complete and that $\varphi_{t}$ is an automorphism of $G$ for all $t \in \mathbb{R}$.

$(2) \Rightarrow(1)$. Let $\left(\varphi_{t}, t \in \mathbb{R}\right)$ be a one parameter group of automorphisms of $G$, and $\mathcal{X}$ its infinitesimal generator. For all right invariant vector fields $Y$, we have

$$
[\mathcal{X}, Y]_{e}=\frac{\mathrm{d}}{\mathrm{d} t}{ }_{\mid t=0} T_{\varphi_{t}(e)} \varphi_{-t} . Y_{\varphi_{t}(e)}=\frac{\mathrm{d}}{\mathrm{d} t}{ }_{t=0} T_{e} \varphi_{-t} . Y_{e}
$$


since $\varphi_{t}(e)=e$ for all $t \in \mathbb{R}$. Considering $\varphi_{-t} \circ R_{\varphi_{t}(x)}=R_{x} \circ \varphi_{-t}$ we have at any point $x$ :

$$
\begin{aligned}
{[\mathcal{X}, Y]_{x} } & =\frac{\mathrm{d}}{\mathrm{d} t} \mid t=0 \\
& =\frac{\mathrm{d}}{\mathrm{d} t} T_{\varphi_{t}(x)} T_{\varphi_{t}(x)} \varphi_{-t} . Y_{\varphi_{t}(x)} T_{e} R_{\varphi_{t}(x)} . Y_{e} \\
& =\frac{\mathrm{d}}{\mathrm{d} t} \mid t=0 T_{e} R_{x} T_{e} \varphi_{-t} . Y_{e} \\
& =T_{e} R_{x} \cdot[\mathcal{X}, Y]_{e} .
\end{aligned}
$$

The vector field $\mathcal{X}$ is therefore affine, and consequently linear since $\varphi_{t}(e)=e$ for all $t \in \mathbb{R}$.

Notations. Here and in the following the flow of a linear vector field $\mathcal{X}$ will be denoted by $\left(\varphi_{t}\right)_{t \in \mathbb{R}}$.

To a given linear vector field $\mathcal{X}$, one can associate the derivation $D$ of $\mathfrak{g}$ defined by:

$$
\forall Y \in \mathfrak{g} \quad D Y=-[\mathcal{X}, Y],
$$

that is $D=-\operatorname{ad}(\mathcal{X})$. The minus sign in this definition comes from the formula $[A x, b]=-A b$ in $\mathbb{R}^{n}$. It also enables to avoid a minus sign in the equality

$$
\forall Y \in \mathfrak{g} \quad \forall t \in \mathbb{R} \quad \varphi_{t}(\exp Y)=\exp \left(\mathrm{e}^{t D} Y\right),
$$

stated in the forthcoming Proposition 3.2.

\section{Example: the inner derivations}

Let $X \in \mathfrak{g}$ be a right invariant vector field. We denote by $\mathcal{I}$ the diffeomorphism of $G$ defined by $x \longmapsto x^{-1}$. The vector field $\mathcal{I}_{*} X$ is left invariant and equal to $-X_{e}$ at $e$. Therefore the vector field

$$
\mathcal{X}=X+\mathcal{I}_{*} X
$$

is linear. Indeed $\mathcal{X}$ belongs to $\mathcal{N}$, because for all $Y \in \mathfrak{g}$, we have $[\mathcal{X}, Y]=\left[X+\mathcal{I}_{*} X, Y\right]=[X, Y] \in \mathfrak{g}$, and satisfies moreover $\mathcal{X}(e)=0$. The derivation associated to $\mathcal{X}$ is inner since it is equal to $-\operatorname{ad}(\mathcal{X})=-\operatorname{ad}(X)(\operatorname{see}$ Sect. 7.1.1 for the example of linear vector fields on matrix Lie groups whose derivation is inner).

This shows also that given an inner derivation $D=-\operatorname{ad}(X)$, there always exists a linear vector field on $G$ whose associated derivation is $D$. This is no longer true in the general case, but holds whenever $G$ is simply connected. This fact is crucial in the sequel.

Theorem 3.2. The group $G$ is assumed to be (connected and) simply connected. Let $D$ be a derivation of its Lie algebra $\mathfrak{g}$. Then there exists one and only one linear vector field on $G$ whose associated derivation is $D$.

Proof. The proof is essentially contained in [3], Lemme 4, p. 250.

Important remark. Under the assumption that the group $G$ is connected, it was stated in Proposition 3.1 that an affine vector field $F$ can be decomposed into $F=\mathcal{X}+Z$, with $\mathcal{X}$ linear and $Z$ left invariant. This decomposition is natural because $\operatorname{ad}(\mathcal{X})=\operatorname{ad}(F)$, but it may be useful to decompose $F$ into a linear part and a right invariant one. Since $\mathcal{I}_{*} Z$ is right invariant and $Z+\mathcal{I}_{*} Z$ linear, we can write

$$
F=\widetilde{\mathcal{X}}-\mathcal{I}_{*} Z \quad \text { with } \widetilde{\mathcal{X}}=\mathcal{X}+Z+\mathcal{I}_{*} Z .
$$

Of course $\operatorname{ad}(\widetilde{\mathcal{X}})=\operatorname{ad}(\mathcal{X})+\operatorname{ad}\left(\mathcal{I}_{*} Z\right) \neq \operatorname{ad}(\mathcal{X})=\operatorname{ad}(F)$ (except if $Z$ belongs to the center of $\left.\mathfrak{g}\right)$.

This remark will be used in Section 4.

Proposition 3.2. For all $t \in \mathbb{R}$

$$
T_{e} \varphi_{t}=\mathrm{e}^{t D}
$$

and consequently

$$
\forall Y \in \mathfrak{g} \quad \forall t \in \mathbb{R} \quad \varphi_{t}(\exp Y)=\exp \left(\mathrm{e}^{t D} Y\right) .
$$


Proof.

(1) Let us first prove the equality

$$
\frac{\mathrm{d}}{\mathrm{d} t} T_{e} \varphi_{t} . Y_{e}=D T_{e} \varphi_{t} . Y_{e} .
$$

This equality has been previously stated at $t=0$ (see equality (3.3) in the proof of Thm. 3.1). In general

$$
\begin{aligned}
\frac{\mathrm{d}}{\mathrm{d} t} T_{e} \varphi_{t} \cdot Y_{e} & =\frac{\mathrm{d}}{\mathrm{d} s} \mid s=0 \\
& =\frac{\mathrm{d}}{\mathrm{d} s} T_{e=0} T_{e} \varphi_{t+s} \cdot Y_{s} \cdot T_{e} \varphi_{t} \cdot Y_{e} \\
& =D T_{e} \varphi_{t} \cdot Y_{e} .
\end{aligned}
$$

(2) From the equality proved above, the first formula of the proposition is immediate. For the second one, remark that $\varphi_{t}$ is a Lie group morphism. Therefore

$$
\begin{aligned}
\varphi_{t}(\exp Y) & =\exp \left(T_{e} \varphi_{t} . Y\right) \\
& =\exp \left(\mathrm{e}^{t D} Y\right) .
\end{aligned}
$$

To finish this section notice the following proposition:

Proposition 3.3. An affine vector field on a connected Lie group is complete.

Proof. This proposition is a consequence of the forthcoming Theorem 5.1, but it can be proved in an elementary way. Indeed let $F$ be an affine vector field and $\mathcal{X}+Z$ its decomposition into a linear vector field $\mathcal{X}$ and a left invariant one $Z$. Let us denote by $t \longmapsto e(t)$ the maximal trajectory of $F$ through the identity $e$, defined on an interval $] a, b[$. One can verify, using the third characterization of linear vector fields (see Thm. 3.1), that $t \longmapsto \varphi_{t}(x) e(t)$ is the trajectory of $F$ through the point $x \in G$, also defined on $] a, b[$. Let us assume $b<+\infty$ and let us choose $x=e(b / 2)$. Then

$$
t \longmapsto \varphi_{t-\frac{b}{2}}\left(e\left(\frac{b}{2}\right)\right) e\left(t-\frac{b}{2}\right)
$$

is the trajectory of $F$ through $x=e(b / 2)$ at $t=b / 2$. Therefore the trajectory $t \longmapsto e(t)$ can be extended up to $3 b / 2$, a contradiction.

\section{Affine VeCtor fields on homogeneous SPACES}

Let $H$ be a closed subgroup of $G$. The homogeneous space $G / H$ is the manifold of left cosets of $H$, and we denote by $\Pi$ the projection of $G$ onto $G / H$. For each right invariant vector field $Y \in \mathfrak{g}$, the projection $\Pi_{*} Y$ of $Y$ onto $G / H$ is always well defined, and will be referred to as an invariant vector field on $G / H$. It is well known that the set of such vector fields, $\Pi_{*} \mathfrak{g}=\left\{\Pi_{*} Y ; Y \in \mathfrak{g}\right\}$, is a Lie algebra and that $\Pi_{*}$ is a Lie algebra morphism from $\mathfrak{g}$ onto $\Pi_{*} \mathfrak{g}$.

Let $\mathcal{X}$ be a linear vector field on $G$. We investigate the existence on $G / H$ of a vector field $\Pi$-related to $\mathcal{X}$. Such a vector field exists if and only if

$$
\forall x \in G, \forall y \in H, \forall t \in \mathbb{R} \quad \Pi\left(\varphi_{t}(x y)\right)=\Pi\left(\varphi_{t}(x)\right) .
$$

But $\Pi\left(\varphi_{t}(x y)\right)=\varphi_{t}(x) \varphi_{t}(y) H$, and the preceding condition is equivalent to

$$
\forall y \in H, \forall t \in \mathbb{R} \quad \varphi_{t}(y) \in H .
$$

Thus $\mathcal{X}$ is $\Pi$-related to a vector field on $G / H$ if and only if $H$ is invariant under the flow of $\mathcal{X}$, therefore if and only if $\mathcal{X}$ is tangent to $H$. 
In the particular case where $H$ is a discrete subgroup of $G$, this amounts to the condition that $\mathcal{X}$ vanishes everywhere on $H$, or that $H$ is included in the set of zeroes of $\mathcal{X}$.

Assume now $H$ to be connected, and denote by $\mathfrak{h}$ its Lie algebra. Since the elements of $H$ are products of exponentials the invariance of $H$ under $\mathcal{X}$ writes

$$
\forall Y \in \mathfrak{h}, \forall t \in \mathbb{R} \quad \varphi_{t}(\exp Y)=\exp \left(\mathrm{e}^{t D} Y\right) \in H
$$

This is equivalent to $\forall Y \in \mathfrak{h}, \forall t \in \mathbb{R}, \mathrm{e}^{t D} Y \in \mathfrak{h}$, and finally to the invariance of $\mathfrak{h}$ under $D$.

Proposition 4.1. Let $H$ be a closed subgroup of $G, G / H$ the homogeneous space of left cosets of $H$, and $\Pi$ the projection of $G$ onto $G / H$.

$A$ linear vector field $\mathcal{X}$ on $G$ is $\Pi$-related to a vector field on $G / H$ if and only if $H$ is invariant under $\mathcal{X}$.

If $H$ is discrete, this condition holds if and only if $H$ is included in the set of zeroes of $\mathcal{X}$.

If $H$ is connected it is equivalent to the invariance of its Lie algebra $\mathfrak{h}$ under the derivation $D$ associated to $\mathcal{X}$.

Under these conditions, the projection of $\mathcal{X}$ onto $G / H$ will be denoted by $\Pi_{*} \mathcal{X}$.

Let us now consider an affine vector field $F$ on $G$. It is equal to

$$
F=\mathcal{X}+Y
$$

where $\mathcal{X}$ is linear and $Y$ right invariant. This decomposition (see "important remark" in Sect. 3) is chosen in order to ensure that the projection $\Pi_{*} Y$ of $Y$ onto $G / H$ is well defined. Then $F$ is $\Pi$-related to a vector field on $G / H$ if and only if $\Pi_{*} \mathcal{X}$ exists. In that case $\Pi_{*} F=\Pi_{*} \mathcal{X}+\Pi_{*} Y$ will stand for the projection of $F$ onto $G / H$.

Proposition 4.2. Let $H$ be a closed subgroup of $G, G / H$ the homogeneous space of left cosets of $H$, and $\Pi$ the projection of $G$ onto $G / H$.

Let $F$ be an affine vector field on $G$ and $F=\mathcal{X}+Y$ its decomposition into a linear vector field $\mathcal{X}$ and a right invariant one $Y$.

Then $F$ is $\Pi$-related to a vector field on $G / H$ if and only if this holds for $\mathcal{X}$, hence if and only if $H$ is invariant under $\mathcal{X}$.

The next task is to define and characterize affine vector fields on connected homogeneous spaces. In general a homogeneous space is defined as a manifold on which a Lie group acts smoothly and transitively. For our purpose it is more convenient to use the following equivalent definition: a (connected) homogeneous space $M$ is a manifold diffeomorphic to a quotient $G / H$, where $G$ is a (connected) Lie group and $H$ a closed subgroup of $G$.

There are two remarks to make about the choice of the Lie group $G$.

(1) We can assume $G$ to be simply connected. If not let $\widetilde{G}$ be the universal covering of $G, \sigma$ the projection of $\widetilde{G}$ onto $G$ and $\widetilde{H}=\sigma^{-1}(H)$. Then $G / H$ is diffeomorphic to $\widetilde{G} / \widetilde{H}$.

(2) We can assume that $\operatorname{dim} \Pi_{*} \mathfrak{g}=\operatorname{dim} \mathfrak{g}$. If not let $\mathfrak{k}$ be the kernel of $\Pi_{*}$ and let $K$ be the connected Lie subgroup of $G$ whose Lie algebra is $\mathfrak{k}$. The subgroup $K$ is normal and included in $H$ because $\mathfrak{k}$ is an ideal of $\mathfrak{g}$ included in the Lie algebra $\mathfrak{h}$ of $H$ (it is easy to see that $Y \in \mathfrak{k}$ if and only if $\forall x \in G, \forall t \in \mathbb{R}$, $\left.x^{-1} \exp (t Y) x \in H\right)$. Now $G / K$ is a simply connected (because $K$ is connected) Lie group and

$$
G / H \sim(G / K) /(H / K)
$$

We can therefore restrict ourselves to homogeneous spaces $G / H$ where $G$ is simply connected and $H$ is a closed subgroup of $G$ such that

$$
\operatorname{dim} \Pi_{*} \mathfrak{g}=\operatorname{dim} \mathfrak{g} .
$$

Notice that whenever the subgroup $H$ is not normal then the projections of the right invariant vector fields belonging to the Lie algebra $\mathfrak{h}$ of $H$ do not vanish in general. More accurately they vanish at the point $H$, 
but not necessarily on $G / H$ (see the second item in the proof of Thm. 4.1). Therefore the dimension of $G / H$ may be strictly less than the dimension of its Lie algebra of invariant vector fields, as shown for instance by Examples 7.2 and 7.3.

Definition 4.1. Let $G$ be a simply connected Lie group, and $H$ a closed subgroup of $G$ such that $\operatorname{dim} \Pi_{*} \mathfrak{g}=$ $\operatorname{dim} \mathfrak{g}$, where $\Pi$ stands for the projection of $G$ onto $G / H$. A vector field $f$ on $G / H$ is said to be affine if it is $\Pi$-related to an affine vector field of $G$.

It is clear that $\Pi_{*} \mathfrak{g}$ is invariant for the Lie bracket with any affine vector field $\Pi_{*} F$. Let us now state and prove the converse statement, and thus characterize the affine vector fields on homogeneous spaces.

Theorem 4.1. Let $G$ be a (connected and) simply connected Lie group, and $H$ a closed subgroup of $G$ such that $\operatorname{dim} \Pi_{*} \mathfrak{g}=\operatorname{dim} \mathfrak{g}$, where $\Pi$ stands for the projection of $G$ onto $G / H$.

$A$ vector field $f$ on $G / H$ is affine if and only if

$$
\forall Y \in \mathfrak{g} \quad\left[f, \Pi_{*} Y\right] \in \Pi_{*} \mathfrak{g},
$$

that is if and only if $\Pi_{*} \mathfrak{g}$ is ad(f)-invariant.

Proof. The necessary part being clear, let us prove the converse and, for this purpose, let us begin by some preliminary remarks.

(1) We can assume without loss of generality $f(H)=0$ since we can add to $f$ an invariant vector field $\Pi_{*} Y$ that verifies $\Pi_{*} Y(H)=-f(H)$.

(2) Let us notice that a right invariant vector field $Y$ belongs to the Lie algebra $\mathfrak{h}$ of $H$ if and only if $\Pi_{*} Y(H)=0$. Indeed

$$
\begin{aligned}
Y \in \mathfrak{h} & \Longleftrightarrow \forall t \in \mathbb{R} \quad \exp (t Y) \in H \\
& \Longleftrightarrow \forall t \in \mathbb{R} \quad \exp (t Y) H=H \\
& \Longleftrightarrow \Pi_{*} Y(H)=0 .
\end{aligned}
$$

By assumption $f$ induces a derivation $\widetilde{D}$ on $\Pi_{*} \mathfrak{g}$, defined by $\widetilde{D}=-\operatorname{ad}(f)$. Since $\mathfrak{g}$ and $\Pi_{*} \mathfrak{g}$ are isomorphic, we can define the derivation $D$ on $\mathfrak{g}$ by the equality $\Pi_{*} \circ D=\widetilde{D} \circ \Pi_{*}$. Moreover $G$ being simply connected there exists a (unique) linear vector field $\mathcal{X}$ on $G$ associated to $D$.

Let $Y \in \mathfrak{h}$. We have

$$
\Pi_{*}[Y, \mathcal{X}]=\Pi_{*}(D Y)=\widetilde{D}\left(\Pi_{*} Y\right)=\left[\Pi_{*} Y, f\right] .
$$

But $\Pi_{*} Y(H)=0$ because $Y \in \mathfrak{h}$, and by assumption $f(H)=0$. Therefore $\left[\Pi_{*} Y, f\right](H)=0$, and $[Y, \mathcal{X}]$ belongs to $\mathfrak{h}$.

This proves that $\mathfrak{h}$ is invariant under $D$. Let $H_{0}$ denote the connected component of $e$ in $H$. Following Proposition 4.1, the linear vector field $\mathcal{X}$ is related to a vector field $\widetilde{f}$ on $G / H_{0}$ by the projection of $G$ onto $G / H_{0}$.

If $H_{0}=H$, then $\widetilde{f}=f$. Indeed $\widetilde{f}$ and $f$ verify

(i) $\forall g \in \Pi_{*} \mathfrak{g} \quad[\widetilde{f}, g]=[f, g]$

(ii) $\widetilde{f}(H)=f(H)=0$,

and according to the forthcoming Lemma 4.1 this implies the equality of $\tilde{f}$ and $f$.

If $H$ is not connected then $G / H_{0}$ is a covering space of $G / H$, and $f$ can be lift to a vector field $f^{\prime}$ on $G / H_{0}$. By the previous method we obtain $\widetilde{f}=f^{\prime}$. Moreover $f$ is related to $f^{\prime}$ by the projection of $G / H_{0}$ onto $G / H$, and therefore to $\mathcal{X}$. More accurately the equality between $\widetilde{f}$ and $f^{\prime}$ implies that $\widetilde{f}$ vanishes on $H / H_{0}$, because so does $f^{\prime}$. Therefore the connected components of $H$ are invariant under $\mathcal{X}$, and according to Proposition 4.1, $\mathcal{X}$ is $\Pi$-related to a vector field on $G / H$ which is nothing else than $f$. 
Lemma 4.1. Let $\left(g_{i}\right)_{i \in I}$ be a transitive family of vector fields (see Sect. 2) on a connected manifold $M$, and let $f$ be a vector field on $M$ that satisfies

$$
\begin{array}{lr}
\text { (i) } \forall i \in I & {\left[f, g_{i}\right]=0} \\
\text { (ii) } \exists x_{0} \in M & f\left(x_{0}\right)=0 .
\end{array}
$$

Then $f=0$.

Proof. Let $\varphi_{t}^{i}$ denote the flow of $g_{i}$. Let $x \in M$. By assumption there exist $i_{1}, \ldots, i_{r} \in I$ and $t_{1}, \ldots, t_{r} \in \mathbb{R}$ such that

$$
x=\varphi_{t_{r}}^{i_{r}} \circ \ldots \circ \varphi_{t_{1}}^{i_{1}}\left(x_{0}\right) .
$$

The flow $\psi_{t}$ of $f$ commutes with $\varphi_{t}^{i}, \forall i \in I$. Hence for all $t$ sufficiently small

$$
\begin{aligned}
\psi_{t}(x) & =\varphi_{t_{r}}^{i_{r}} \circ \ldots \circ \varphi_{t_{1}}^{i_{1}}\left(\psi_{t}\left(x_{0}\right)\right) \\
& =\varphi_{t_{r}}^{i_{r}} \ldots \circ \varphi_{t_{1}}^{i_{1}}\left(x_{0}\right) \\
& =x
\end{aligned}
$$

Therefore $f(x)=0$.

Example. The affine vector fields on the sphere $S^{n}$ are described in Section 7.1.2 and this example is generalized in Section 7.1.3.

The next proposition is obvious but useful in the sequel.

Proposition 4.3. An affine vector field on a homogeneous space is complete.

We can now state the definition of general linear systems. They are the systems

$$
\dot{x}=F(x)+\sum_{j=1}^{m} u_{j} Y_{j}(x)
$$

on homogeneous spaces $G / H$, where the field $F$ is affine and the $Y_{j}$ 's invariant. Linear systems on Lie groups, obtained when the subgroup $H$ is normal, and invariant systems, obtained when the vector field $F$ is invariant, are two particular cases of this general setting.

\section{Finite dimensional Algebras of VeCtor Fields}

Let $\Gamma=\left\{g_{i} ; i \in I\right\}$ be a family of vector fields on a connected manifold $M$. All the vector fields $g_{i}$ belonging to $\Gamma$ are assumed to be $\mathcal{C}^{k}$, for a common $k \geq 1$, and $M$ is therefore at least $\mathcal{C}^{k+1}$.

Recall that the family $\Gamma$ is said to generate a Lie algebra if all the Lie brackets of elements of $\Gamma$ of all finite lengths exist and are also $\mathcal{C}^{k}$, and that we define in that case the Lie algebra $\mathcal{L}(\Gamma)$ as the subspace of $V^{k}(M)$ spanned by these Lie brackets.

Theorem 5.1. Let $\Gamma$ be a family of $\mathcal{C}^{k}$ vector fields on a connected manifold $M$. If

(i) all the vector fields belonging to $\Gamma$ are complete;

(ii) $\Gamma$ generates a finite dimensional Lie algebra $\mathcal{L}(\Gamma)$;

(iii) the family $\Gamma$ is transitive;

then $M$ is $\mathcal{C}^{k+1}$ diffeomorphic to a homogeneous space $G / H$, where $G$ is a (connected and) simply connected Lie Group whose Lie algebra is isomorphic to $\mathcal{L}(\Gamma)$, and $H$ is a closed subgroup of $G$.

By this diffeomorphism $\mathcal{L}(\Gamma)$ is related to the Lie algebra of invariant vector fields on $G / H$, and $\Gamma$ to a subset of this Lie algebra.

Moreover all the vector fields belonging to $\mathcal{L}(\Gamma)$ are complete. 
(1) Let $G$ be a (connected and) simply connected Lie group whose Lie algebra $\mathfrak{g}$ is isomorphic to $\mathcal{L}(\Gamma)$, $\mathcal{L}$ in short, and let us denote by

$$
L: \quad \mathcal{L} \longrightarrow \mathfrak{g}
$$

this Lie algebra isomorphism.

(2) In the product $M \times G$ consider the distribution spanned by the family of vector fields $\{(g, L(g)) ; g \in \mathcal{L}\}$. This distribution is involutive, and its rank is constant, equal to $\operatorname{dim}(G)=\operatorname{dim}(\mathcal{L})$. Henceforth it is completely integrable (the proof of the Frobenius theorem for $\mathcal{C}^{k}$ vector fields on a $\mathcal{C}^{k+1}$ manifold with $k \geq 1$ can be found for instance in [8]).

Let us fix an arbitrary point $p_{0}$ in $M$, and let $S$ be the leaf of the foliation through the point $\left(p_{0}, e\right)$ (where $e$ stands for the identity element of $G$ ). We denote by $\Pi_{1}$ (resp. $\Pi_{2}$ ) the projection of $S$ onto $M$ (resp. onto $G$ ). We are going to prove that $\Pi_{2}$ is a diffeomorphism.

(3) Notations. For $g_{i} \in \Gamma=\left\{g_{i} ; i \in I\right\}$ we denote by $Y_{i}=L\left(g_{i}\right)$ the corresponding vector field on $G$. The flows of $g_{i}$ and $Y_{i}$ are respectively denoted by

$$
(t, p) \longmapsto \gamma_{t}^{i}(p) \text { and }(t, x) \longmapsto \exp \left(t Y_{i}\right) x .
$$

(4) First of all let us show that $\Pi_{2}$ is onto. Since $L$ is a Lie algebra isomorphism and $\Gamma$ generates $\mathcal{L}$, the Lie algebra $\mathfrak{g}$ of $G$ is generated by the family $L(\Gamma)=\left\{Y_{i} ; i \in I\right\}$. This family is therefore transitive on $G$ which is connected. Let $x \in G$. There exists an integer $r$, indices $i_{1}, \ldots, i_{r} \in I$ and real numbers $t_{1}, \ldots, t_{r}$ such that

$$
x=\exp \left(t_{r} Y_{i_{r}}\right) \ldots \exp \left(t_{1} Y_{i_{1}}\right) .
$$

Thanks to the completeness assumption of the elements of $\Gamma$ the point

$$
p=\gamma_{t_{r}}^{i_{r}} \circ \ldots \circ \gamma_{t_{1}}^{i_{1}}\left(p_{0}\right)
$$

is well defined. Moreover the vector fields $\left(g_{i}, Y_{i}\right)$ are tangent to $S$ and the point $(p, x)$ belongs to $S$. Its projection onto $G$ is $x$, and this proves the surjectivity of $\Pi_{2}$.

(5) Let us now prove that $\Pi_{2}$ is a covering map. Since the family $L(\Gamma)=\left\{Y_{i} ; i \in I\right\}$ generates the Lie algebra $\mathfrak{g}$ of $G$, the identity $e$ is normally accessible from $e$ (for the family $\left\{ \pm Y_{i} ; i \in I\right\}$ ) (see [7] for the notion of normal accessibility, in particular Cor. 1, p. 154). Let $n$ be the dimension of $G$. We can find indices $i_{1}, \ldots, i_{n} \in I$ and real numbers $t_{1}, \ldots, t_{n}>0$ such that the mapping

$$
\left(s_{1}, \ldots, s_{n}\right) \longmapsto \exp \left(\left(t_{n}+s_{n}\right) Y_{i_{n}}\right) \ldots \exp \left(\left(t_{1}+s_{1}\right) Y_{i_{1}}\right)
$$

is a local diffeomorphism at $(0, \ldots, 0) \in \mathbb{R}^{n}$.

Let $x_{0}=\exp \left(-t_{1} Y_{i_{1}}\right) \ldots \exp \left(-t_{n} Y_{i_{n}}\right)$. Then the mapping $\Psi$ defined by

$$
\left(s_{1}, \ldots, s_{n}\right) \longmapsto \exp \left(\left(t_{n}+s_{n}\right) Y_{i_{n}}\right) \ldots \exp \left(\left(t_{1}+s_{1}\right) Y_{i_{1}}\right) x_{0}
$$

is also a local diffeomorphism, and satisfies $\Psi(0, \ldots, 0)=e$.

We can choose a neighbourhood of 0 in $\mathbb{R}^{n}$ sent diffeomorphically by $\Psi$ onto an open and connected neighbourhood $V$ of $e$ in $G$. Let us denote by $\tilde{\Psi}$ the similar mapping from $\mathbb{R}^{n} \times M$ into $M$, that is

$$
\left(s_{1}, \ldots, s_{n} ; p\right) \longmapsto \gamma_{t_{n}+s_{n}}^{i_{n}} \circ \ldots \circ \gamma_{t_{1}+s_{1}}^{i_{1}} \circ \gamma_{-t_{1}}^{i_{1}} \circ \ldots \circ \gamma_{-t_{n}}^{i_{n}}(p) .
$$

Let $x$ be a given point in $G$, and for every $p \in M$ such that $(p, x) \in S$ let $\sigma_{p}$ be the mapping

$$
\begin{array}{rlc}
\sigma_{p}: \quad V x & \longrightarrow & S \\
y & \longmapsto(\tilde{\Psi}(\tau)(p), y)
\end{array}
$$


where $\tau=\Psi^{-1}\left(y x^{-1}\right)$. The neighbourhood $V x$ of $x$ is evenly covered by $\left\{\sigma_{p}(V x) ; p \in M\right.$ and $(p, x) \in S\}$. Indeed it is clear that $\Pi_{2} \circ \sigma_{p}$ is the identity of $V x$ and that the sets $\sigma_{p}(V x)$ cover $\Pi_{2}^{-1}(V x)$. Let us show that they are mutually disjoint. If not we can find $\tau, \tau^{\prime}, p, p^{\prime}$, such that $\sigma_{p}(\Psi(\tau) . x)=\sigma_{p^{\prime}}\left(\Psi\left(\tau^{\prime}\right) \cdot x\right)$. But

$$
\begin{aligned}
\sigma_{p}(\Psi(\tau) \cdot x)=\sigma_{p^{\prime}}\left(\Psi\left(\tau^{\prime}\right) \cdot x\right) & \Longleftrightarrow(\tilde{\Psi}(\tau)(p), \Psi(\tau) \cdot x)=\left(\tilde{\Psi}\left(\tau^{\prime}\right)\left(p^{\prime}\right), \Psi\left(\tau^{\prime}\right) \cdot x\right) \\
& \Longrightarrow \Psi(\tau) \cdot x=\Psi\left(\tau^{\prime}\right) \cdot x \\
& \Longrightarrow \tau=\tau^{\prime} \\
& \Longrightarrow \tilde{\Psi}(\tau)(p)=\tilde{\Psi}(\tau)\left(p^{\prime}\right) \\
& \Longrightarrow p=p^{\prime} .
\end{aligned}
$$

This proves that $\Pi_{2}$ is a covering of $G$ by $S$ (notice that $S$ is connected and locally connected). But $G$ is simply connected and $\Pi_{2}$ is therefore a diffeomorphism. In particular, given a point $x \in G$, there is one and only one point $p \in M$ for which $(p, x) \in S$.

(6) The next task is to prove that the left translations of $G$ induce a group action on $M$. Let $y \in G$ and $(p, x) \in S$. There exists an unique point $q \in M$ such that $(q, y x)$ belongs to $S$. Let us show that $q$ depends only on $p$ and $y$ but not on a particular choice of $x$. Thanks again to the transitivity of the family $L(\Gamma)$, there exist an integer $r$, indices $i_{1}, \ldots, i_{r} \in I$ and real numbers $t_{1}, \ldots, t_{r}$ such that

$$
y=\exp \left(t_{r} Y_{r}\right) \ldots \exp \left(t_{1} Y_{1}\right)
$$

Then the equality $q=\gamma_{t_{r}}^{i_{r}} \circ \ldots \circ \gamma_{t_{1}}^{i_{1}}(p)$ holds.

Indeed $\left(\gamma_{t_{r}}^{i_{r}} \circ \ldots \circ \gamma_{t_{1}}^{i_{1}}(p), \exp \left(t_{r} Y_{r}\right) \ldots \exp \left(t_{1} Y_{1}\right) x\right)$ belongs to $S$, and $\gamma_{t_{r}}^{i_{r}} \circ \ldots \circ \gamma_{t_{1}}^{i_{1}}(p)$ is therefore the only point $q$ such that $\left(q, \exp \left(t_{r} Y_{r}\right) \ldots \exp \left(t_{1} Y_{1}\right) x\right)=(q, y x)$ belongs to $S$.

We will denote by $\rho_{y}$ the diffeomorphism $\gamma_{t_{r}}^{i_{r}} \circ \ldots \circ \gamma_{t_{1}}^{i_{1}}$ of $M$. Notice that

$$
\left(\rho_{y}(p), y x\right)=\Pi_{2}^{-1} \circ L_{y} \circ \Pi_{2}(p, x) .
$$

Hence the mapping

$$
(y, p) \longmapsto \rho_{y}(p)
$$

is of class $\mathcal{C}^{k+1}$ from $G \times M$ onto $M$.

To finish we have $\rho_{y} \circ \rho_{y^{\prime}}=\rho_{y y^{\prime}}$ for all $y, y^{\prime} \in G$, according to equality (5.1).

Therefore $(y, p) \longmapsto \rho_{y}(p)$ is a transitive and $\mathcal{C}^{k+1}$ action of the Lie group $G$ on the manifold $M$. Let $H$ be the isotropy group of $p_{0}$, that is the set of points $x$ of $G$ such that $\left(p_{0}, x\right)$ belongs to $S$, and $G / H$ the manifold of left cosets of $H$. Then

$$
x H \in G / H \longmapsto \rho_{x}\left(p_{0}\right)
$$

is a diffeomorphism from $G / H$ onto $M$, denoted by $\Phi$ in the end of the proof.

(7) We are left to prove that $\Phi$ induces an isomorphism between the Lie algebra of invariant vector fields on $G / H$ and $\mathcal{L}$. Let us denote by $\Pi$ the projection of $G$ onto $G / H$. Then the equality

$$
\Phi \circ \Pi=\Pi_{1} \circ \Pi_{2}^{-1}
$$

holds. Recall that $\left(p_{0}, e\right) \in S$. Then $\forall x \in G$

$$
\begin{aligned}
\Phi \circ \Pi(x) & =\Phi(x H)=\rho_{x}\left(p_{0}\right) \\
& =\Pi_{1}\left(\rho_{x}\left(p_{0}\right), x\right) \\
& =\Pi_{1} \circ \Pi_{2}^{-1}(x) .
\end{aligned}
$$


Let $Y \in \mathfrak{g}$. Then $\Phi_{*}\left(\Pi_{*} Y\right)=\left(\Pi_{1} \circ \Pi_{2}^{-1}\right)_{*} Y=L^{-1}(Y)$. This equality has two consequences. The first one is that $\Gamma$ is under $\Phi^{-1}$ equivalent to a set of invariant vector fields on $G / H$. The second one is that all the vector fields of $\mathcal{L}(\Gamma)$ are complete, since $\Phi$ is a diffeomorphism, and they are related by $\Phi_{*}$ to complete vector field of $G / H$.

Thanks to the Orbit Theorem, recalled in Section 2, we can relax the transitivity assumption, and obtain the following corollary.

Corollary 5.1. Let $\Gamma$ be a family of $\mathcal{C}^{k}$ vector fields on a connected manifold $M$. If

(i) all the vector fields belonging to $\Gamma$ are complete;

(ii) $\Gamma$ generates a finite dimensional Lie algebra $\mathcal{L}(\Gamma)$,

then all the vector fields belonging to $\mathcal{L}(\Gamma)$ are complete, and the family $\Gamma$ is Lie-determined.

Proof. Let $p$ be a point of $M$ and let us denote by $S$ the orbit of $\Gamma$ through $p$. By the orbit theorem $S$ is a submanifold of $M$. Moreover every vector field belonging to $\Gamma$, hence every vector field belonging to $\mathcal{L}(\Gamma)$, is tangent to $S$.

Let $\Gamma_{S}$ stand for the family of restrictions to $S$ of the vector fields of $\Gamma$. Clearly $\Gamma_{S}$ generates a finite dimensional Lie algebra $\mathcal{L}\left(\Gamma_{S}\right)$, which is nothing else than the set of restrictions to $S$ of the vector fields of $\mathcal{L}(\Gamma)$. By definition the family $\Gamma_{S}$ is transitive on $S$ and satisfies the assumptions of Theorem 5.1. Therefore $S$ is diffeomorphic to a homogeneous space $G / H$, where $G$ is a simply connected Lie Group whose Lie algebra is isomorphic to $\mathcal{L}\left(\Gamma_{S}\right)$, and $H$ is a closed subgroup of $G$. By this diffeomorphism $\Gamma_{S}$ is related to a set of invariant vector fields, and $\mathcal{L}\left(\Gamma_{S}\right)$ to the Lie algebra of invariant vector fields on $G / H$. Therefore

$$
\forall q \in S \quad \operatorname{rank} \mathcal{L}\left(\Gamma_{S}\right)(q)=\operatorname{dim} G / H=\operatorname{dim} S
$$

and the family $\Gamma_{S}$ is Lie-determined.

\section{Application to CONTROL Systems}

Consider the control affine system

$$
\dot{x}=f(x)+\sum_{j=1}^{m} u_{j} g_{j}(x)
$$

where $x$ belongs to the $n$-dimensional connected manifold $M$ and where $f, g_{1}, \ldots, g_{m}$ are $\mathcal{C}^{k}$ vector fields on $M$, with $k \geq 1$. The control $u=\left(u_{1}, \ldots, u_{m}\right)$ belongs to $\mathbb{R}^{m}$.

The family $\Gamma=\left\{f, g_{1}, \ldots, g_{m}\right\}$ is assumed to generate a Lie algebra denoted by $\mathcal{L}$.

We also denote by $\mathcal{L}_{0}$ the ideal of $\mathcal{L}$ generated by $g_{1}, \ldots, g_{m}$. It is well known that $\mathcal{L}_{0}$ is the smallest Lie subalgebra of $\mathcal{L}$ containing $g_{1}, \ldots, g_{m}$ and closed for the Lie bracket with $f$ :

$$
X \in \mathcal{L}_{0} \quad \Longrightarrow \quad[f, X] \in \mathcal{L}_{0}
$$

The dimension of $\mathcal{L}_{0}$ is its dimension as a real Lie algebra, and its rank at a point $p \in M$ is the dimension of the subspace $\left\{X(p) ; X \in \mathcal{L}_{0}\right\}$ of the tangent space $T_{p} M$ of $M$ at $p$. In the particular case where the rank of $\mathcal{L}_{0}$ is constant, it will be referred to as rank $\left(\mathcal{L}_{0}\right)$.

It is also known that $\mathcal{L}=\mathcal{L}_{0}+\mathbb{R} f$. If the point $p$ is a zero of $f$ then $\operatorname{rank}(\mathcal{L})(p)=\operatorname{rank}\left(\mathcal{L}_{0}\right)(p)$, and in case where the ranks of $\mathcal{L}$ and $\mathcal{L}_{0}$ are constant, the existence of a zero of $f$ implies their equality.

If $\mathcal{L}$ (resp. $\left.\mathcal{L}_{0}\right)$ is finite dimensional, then $G$ (resp. $G_{0}$ ) will stand for a (connected and) simply connected Lie group whose Lie algebra is isomorphic to $\mathcal{L}\left(\operatorname{resp} . \mathcal{L}_{0}\right)$. 
Theorem 6.1. We assume the family $\left\{f, g_{1}, \ldots, g_{m}\right\}$ to be transitive. Then system $(\Sigma)$ is diffeomorphic to a linear system on a Lie group or a homogeneous space if and only if the vector fields $f, g_{1}, \ldots, g_{m}$ are complete and generate a finite dimensional Lie algebra.

More accurately, under this condition the rank of $\mathcal{L}_{0}$ is constant, equal to $\operatorname{dim}(M)$ or $\operatorname{dim}(M)-1$, and:

(i) if rank $\left(\mathcal{L}_{0}\right)=\operatorname{dim}(M)$, in particular if there exists one point $p_{0} \in M$ such that $f\left(p_{0}\right)=0$, then $(\Sigma)$ is diffeomorphic to a linear system on a homogeneous space $G_{0} / H$ of $G_{0}$;

(ii) if rank $\left(\mathcal{L}_{0}\right)=\operatorname{dim}(M)-1$, then $\Sigma$ is diffeomorphic to an invariant system on a homogeneous space $G / H$ of $G$.

Proof. Let us prove the sufficiency.

The Lie algebras $\mathcal{L}$ and $\mathcal{L}_{0}$ are finite dimensional and generated by complete vector fields. By Corollary 5.1 they are Lie-determined. As $\mathcal{L}$ is transitive, its rank is everywhere full. Moreover the rank of $\mathcal{L}_{0}$ is constant over $M$, equal to $\operatorname{dim}(M)$ or $\operatorname{dim}(M)-1$. Indeed $\mathcal{L}_{0}$ being Lie-determined, its rank is everywhere equal to the dimension of the zero-time orbit, which is constant, equal to $\operatorname{dim}(M)$ or $\operatorname{dim}(M)-1$ (see [7]).

Let us assume rank $\left(\mathcal{L}_{0}\right)=\operatorname{dim}(M)$. Then we can apply Theorem 5.1 to the family $\mathcal{L}_{0}$. The manifold $M$ is diffeomorphic to a homogeneous space $G_{0} / H$ of $G_{0}$, and if we denote by $\Phi$ this diffeomorphism, the tangent mapping $\Phi_{*}$ induces a Lie algebra isomorphism between $\mathcal{L}_{0}$ and the Lie algebra of invariant vector fields on $G_{0} / H$. The vector field $\Phi_{*} f$ satisfies

$$
\forall g \in \mathcal{L}_{0} \quad\left[\Phi_{*} f, \Phi_{*} g\right]=\Phi_{*}[f, g] \in \Phi_{*}\left(\mathcal{L}_{0}\right) .
$$

Since $\Phi_{*}\left(\mathcal{L}_{0}\right)$ is equal to the Lie algebra of invariant vector fields on $G_{0} / H$, and according to Theorem 4.1, the vector field $\Phi_{*} f$ is affine, that is $\Phi_{*} f$ is the projection onto $G_{0} / H$ of an affine vector field $F$ of $G_{0}$. This vector field can be chosen to be linear if and only if there is one point $p_{0}$ in $M$ such that $f\left(p_{0}\right)=0$ : in the proof of Theorem 5.1, we can choose $p_{0}$ to be the projection of the identity $e$ of $G_{0}$. Clearly system $(\Sigma)$ is diffeomorphic to the linear system

$$
\dot{x}=F(x)+\sum_{j=1}^{m} u_{j} Y_{j}(x)
$$

on $G_{0} / H$, where $F$ stands for $\Phi_{*} f$, and $Y_{j}=\Phi_{*} g_{j}$ is an invariant vector field for $j=1, \ldots, m$.

We assume now rank $\left(\mathcal{L}_{0}\right)=\operatorname{dim}(M)-1$. We apply Theorem 5.1 to $\mathcal{L}$ : the manifold $M$ is diffeomorphic to a homogeneous space $G / H$ of $G$, and under this diffeomorphism $\mathcal{L}$ is isomorphic to the Lie algebra of invariant vector fields on $G / H$. System $(\Sigma)$ is obviously diffeomorphic to an invariant system on $G / H$.

From Theorem 6.1 we can deduce the following corollary, stated in the $\mathcal{C}^{\infty}$ case in order to ensure the existence of $\mathcal{L}$ and $\mathcal{L}_{0}$ :

Corollary 6.1. The manifold $M$ is assumed to be $\mathcal{C}^{\infty}$ and simply connected.

The family $\left\{f, g_{1}, \ldots, g_{m}\right\}$ is assumed to be $\mathcal{C}^{\infty}$, complete and transitive, and the vector field $f$ to vanish at a point $p_{0} \in M$.

Then system $(\Sigma)$ is equivalent to a linear system on a Lie group if and only if

$$
\operatorname{dim}\left(\mathcal{L}_{0}\right)=\operatorname{dim}(M) .
$$

Proof. The necessity part is obvious. Let us prove the sufficient one.

Since $\operatorname{dim}\left(\mathcal{L}_{0}\right)<\infty$, Theorem 6.1 applies, and since $f$ vanishes at one point, we have $\operatorname{rank}\left(\mathcal{L}_{0}\right)=\operatorname{dim}(M)=$ $\operatorname{dim}\left(\mathcal{L}_{0}\right)$. Therefore $\Sigma$ is diffeomorphic to a linear system on a homogeneous space $G_{0} / H$ of $G_{0}$, with the previous notations. Now the two conditions $\operatorname{dim}\left(\mathcal{L}_{0}\right)=\operatorname{dim}(M)$ and $M$ simply connected imply $G_{0} / H=G_{0}$.

The assumption that $M$ is simply connected cannot be relaxed. If not, $M$ remains diffeomorphic to a homogeneous space $G / H$, where $H$ is discrete, but $G / H$ is a Lie group if and only if $H$ is normal. 


\section{EXAMPLES}

\subsection{Examples of linear and affine vector fields}

\subsubsection{Inner derivations on matrix Lie groups}

See [9]. Let $G$ be a connected matrix Lie group, that is a connected Lie subgroup of $G l(n ; \mathbb{R})$, for some $n$. For any matrix $X$ belonging to the tangent space $T_{I} G$ at the identity $I$, identified to $\mathfrak{g}$, the mapping $M \longmapsto X M$ defines a right invariant vector field. We can also associate to the inner derivation $D=-\operatorname{ad}(X)$ the linear vector field $\mathcal{X}$ defined by

$$
\mathcal{X}(M)=X M-M X \text {. }
$$

Hence in the inner derivation case, a linear system on $G$ writes

$$
\dot{M}=X M-M X+\sum_{j=1}^{m} u_{j} Y_{j} M
$$

where $M \in G$, and $X, Y_{1}, \ldots, Y_{m} \in \mathfrak{g}$.

7.1.2. Affine vector fields on the sphere $S^{n}, n \geq 2$

The sphere $S^{n}$ is diffeomorphic to the homogeneous space $S O_{n+1} / S O_{n}$, where $S O_{n}$ is identified with the closed subgroup

$$
H=\left\{\left(\begin{array}{c|ccc}
1 & 0 & \ldots & 0 \\
\hline 0 & & \\
\vdots & & N
\end{array}\right) ; N \in S O_{n}\right\}
$$

of $S O_{n+1}$.

On the one hand the Lie algebra $\mathfrak{s o}_{n+1}$ is semi simple, since $n+1 \geq 3$, so all its derivations are inner.

On the other hand the subgroup $H$ is connected, and, following Proposition 4.1, a linear vector field $\mathcal{X}$ on $S O_{n+1}$ is related to a vector field on $S O_{n+1} / H$ if and only if its Lie algebra $\mathfrak{h}$ is invariant under $D=-\operatorname{ad}(\mathcal{X})$.

But $\mathcal{X}$ being of the form

$$
\mathcal{X}(M)=X M-M X, \quad M \in S O_{n+1}
$$

for some $X \in \mathfrak{s o}_{n+1}$, this condition turns out to

$$
\forall Y \in \mathfrak{h} \quad[X, Y] \in \mathfrak{h}
$$

and a straightforward computation shows that it holds if and only if $X \in \mathfrak{h}$.

The flow of $\mathcal{X}$ is given by

$$
\varphi_{t}(M)=\mathrm{e}^{t X} M \mathrm{e}^{-t X}
$$

and its projection onto $S O_{n+1} / H$ is equal to

$$
\mathrm{e}^{t X} M \mathrm{e}^{-t X} H=\mathrm{e}^{t X} M H
$$

since $\forall t \in \mathbb{R}, \mathrm{e}^{-t X} \in H$. This proves that the vector fields $\mathcal{X}$ and $X$ have the same projection on $S^{n} \sim S O_{n+1} / H$.

In conclusion the only affine vector fields on the sphere $S^{n}$ are the invariant ones. They are the vector fields defined by

$$
f(x)=A x, \quad x \in S^{n}
$$

where $A \in \mathfrak{s o}_{n+1}$, that is $A^{\prime}=-A$. 
Remark. The group $S O_{n+1}$ is not simply connected but as it is semi simple, this is not a restriction to the application of Theorem 4.1. Indeed any derivation $D$ is inner, hence the associated linear vector field always exists, despite the lack of simply connectedness.

\subsubsection{The general inner derivation case}

The previous phenomena is due to the fact that the normalizer of $\mathfrak{h} \sim \mathfrak{s o}_{n}$ in $\mathfrak{s o}_{n+1}$ is itself. To see this let us consider the general inner derivation case on a connected Lie group $G$.

Let $D=-\operatorname{ad}(X)$, with $X \in \mathfrak{g}$, be an inner derivation. Recall from Section 3 that the linear vector field associated to $D$ is equal to

$$
\mathcal{X}=X+\mathcal{I}_{*} X .
$$

Let $H$ be a closed and connected subgroup of $G$. Then, following again Proposition 4.1, the linear vector field $\mathcal{X}$ is related to a vector field on $G / H$ if and only if its Lie algebra $\mathfrak{h}$ is invariant under $D=-\operatorname{ad}(\mathcal{X})$. But

$$
\begin{aligned}
& \forall Y \in \mathfrak{h} \quad[Y, \mathcal{X}]=[Y, X] \in H \\
& \Longleftrightarrow \quad X \in \text { norm }_{\mathfrak{g}} \mathfrak{h} .
\end{aligned}
$$

Therefore $\mathcal{X}$ can be projected on $G / H$ if and only if $X$ belongs to the normalizer of $\mathfrak{h}$ in $\mathfrak{g}$.

An example of linear vector field whose derivation is not inner is given in the next section.

\subsection{Example of equivalence}

Consider the system in $\mathbb{R}^{2}$

$$
\Sigma=\left\{\begin{array}{ll}
\dot{x} & =y^{2} \\
\dot{y} & =u
\end{array} .\right.
$$

Let

$$
f=y^{2} \frac{\partial}{\partial x}, \quad g_{1}=\frac{\partial}{\partial y}, \quad g_{2}=\left[g_{1}, f\right]=2 y \frac{\partial}{\partial x}, \quad \text { and } g_{3}=\left[g_{1}, g_{2}\right]=2 \frac{\partial}{\partial x} .
$$

Clearly the system can be written

$$
\dot{p}=f(p)+u g(p) \quad \text { where } \quad p=(x, y) .
$$

The brackets $\left[g_{1}, g_{3}\right],\left[g_{2}, g_{3}\right],\left[f, g_{2}\right]$, and $\left[f, g_{3}\right]$ vanish, and with the notations of Section 6 we have

$$
\mathcal{L}=S p\left\{f, g_{1}, g_{2}, g_{3}\right\} \text { and } \mathcal{L}_{0}=S p\left\{g_{1}, g_{2}, g_{3}\right\}
$$

All these vector fields are complete, $\mathcal{L}_{0}$ is isomorphic to the Heisenberg Lie algebra, and its rank is everywhere full, therefore Theorem 6.1 applies: system $(\Sigma)$ is equivalent to a linear system on a homogeneous space of the Heisenberg group.

Let us compute this equivalence. The Heisenberg group is

$$
G=\left\{\left(\begin{array}{ccc}
1 & y & z \\
0 & 1 & x \\
0 & 0 & 1
\end{array}\right) ; \quad(x, y, z) \in \mathbb{R}\right\}
$$

and its Lie algebra $\mathfrak{g}$ is spanned by the right invariant vector fields

$$
X=\left(\begin{array}{lll}
0 & 0 & 0 \\
0 & 0 & 1 \\
0 & 0 & 0
\end{array}\right), \quad Y=\left(\begin{array}{ccc}
0 & 1 & x \\
0 & 0 & 0 \\
0 & 0 & 0
\end{array}\right), \quad \text { and } Z=\left(\begin{array}{lll}
0 & 0 & 1 \\
0 & 0 & 0 \\
0 & 0 & 0
\end{array}\right)
$$


that can be written in the canonical coordinates

$$
X=\frac{\partial}{\partial x}, \quad Y=\frac{\partial}{\partial y}+x \frac{\partial}{\partial z}, \quad \text { and } \quad Z=\frac{\partial}{\partial z} .
$$

The derivation $D$ on $\mathfrak{g}$ should verify $D X=Y$ and $D Y=D Z=0$. Let $\mathcal{X}$ be the unique vector field that verifies $\mathcal{X}(I)=0$ and $-\operatorname{ad}(\mathcal{X})=D$, hence $[X, \mathcal{X}]=Y$ and $[Y, \mathcal{X}]=[Z, \mathcal{X}]=0$. Using indeterminate coefficients, we get

$$
\mathcal{X}=x \frac{\partial}{\partial y}+\frac{1}{2} x^{2} \frac{\partial}{\partial z}
$$

It is easy to check that $\mathcal{X}$ is linear and that $-\operatorname{ad}(\mathcal{X})=D$. The system $\dot{q}=\mathcal{X}(q)+u X(q)$ can be written in $\mathbb{R}^{3}$

$$
\begin{cases}\dot{x} & =u \\ \dot{y} & =x \\ \dot{z} & =\frac{1}{2} x^{2}\end{cases}
$$

We are looking for a subgroup $H$ of $G$ for which $(\Sigma)$ is equivalent to a linear system on $G / H$. The algebra of vector fields of $\mathcal{L}_{0}$ that vanishes at $(0,0)$ is spanned by $g_{2}$, thus the Lie algebra of $H$ should be spanned by $Y$.

Let $H$ be the closed, but not normal, subgroup of $G$

$$
H=\left\{\left(\begin{array}{lll}
1 & y & 0 \\
0 & 1 & 0 \\
0 & 0 & 1
\end{array}\right) ; y \in \mathbb{R}\right\}
$$

The projection of $G$ onto $G / H$ is equivalent to the projection $(x, y, z) \longmapsto(x, z)$ from $\mathbb{R}^{3}$ onto $\mathbb{R}^{2}$, and the linear system on $G / H$ is therefore equivalent to

$$
\Sigma^{\prime}= \begin{cases}\dot{x} & =u \\ \dot{z} & =\frac{1}{2} x^{2}\end{cases}
$$

To finish, $\Sigma^{\prime}$ is equivalent to $(\Sigma)$ under the linear transformation of $\mathbb{R}^{2},(x, z) \longmapsto(2 z, x)$.

\subsection{Generalization of the previous example}

Let $P(y)$ be a polynomial. Then the system in $\mathbb{R}^{2}$

$$
\begin{cases}\dot{x} & =P(y) \\ \dot{y} & =u\end{cases}
$$

satisfies the assumptions of Theorem 6.1: let

$$
f=P(y) \frac{\partial}{\partial x}, \quad g_{1}=\frac{\partial}{\partial y}, \quad g_{2}=\left[g_{1}, f\right]=P^{\prime}(y) \frac{\partial}{\partial x}
$$

and, by induction:

$$
g_{k+1}=\left[g_{1}, g_{k}\right]=P^{(k)}(y) \frac{\partial}{\partial x}
$$

The vector field $g_{k}$ vanishes as soon as $k>\operatorname{deg}(P)+1$, and so do the other brackets. Therefore

$$
\mathcal{L}=S p\left\{f, g_{1}, \ldots, g_{\operatorname{deg}(P)+1}\right\} \text { and } \mathcal{L}_{0}=S p\left\{g_{1}, \ldots, g_{\operatorname{deg}(P)+1}\right\}
$$

All these vector fields are complete, the system satisfies clearly the rank condition, therefore Theorem 6.1 applies. 
Acknowledgements. The author wishes to express his thanks to Professor Witold Respondek for drawing his attention to equivalence problems, and for stimulating conversations.

\section{REFERENCES}

[1] V. Ayala and L. San Martin, Controllability properties of a class of control systems on Lie groups, in Nonlinear control in the year 2000, Vol. 1 (Paris), Lect. Notes Control Inform. Sci. 258, Springer (2001) 83-92.

[2] V. Ayala and J. Tirao, Linear control systems on Lie groups and Controlability, in Proceedings of Symposia in Pure Mathematics, Vol. 64, AMS (1999) 47-64.

[3] N. Bourbaki, Groupes et algèbres de Lie, Chapitres 2 et 3. CCLS, France (1972).

[4] F. Cardetti and D. Mittenhuber, Local controllability for linear control systems on Lie groups. J. Dyn. Control Syst. 11 (2005) 353-373.

[5] G. Hochschild, The Structure of Lie Groups. Holden-Day (1965).

[6] Ph. Jouan, On the existence of observable linear systems on Lie Groups. J. Dyn. Control Syst. 15 (2009) 263-276.

[7] V. Jurdjevic, Geometric control theory. Cambridge University Press (1997).

[8] P. Malliavin, Géométrie différentielle intrinsèque. Hermann, Paris, France (1972).

[9] L. Markus, Controllability of multitrajectories on Lie groups, in Dynamical systems and turbulence, Warwick (1980), Lect. Notes Math. 898, Springer, Berlin-New York (1981) 250-265.

[10] R.S. Palais, A global formulation of the Lie theory of transformation groups, Memoirs of the American Mathematical Society 22. AMS, Providence, USA (1957).

[11] H.J. Sussmann, Orbits of families of vector fields and integrability of distributions. Trans. Amer. Math. Soc. 180 (1973) 171-188. 